\title{
ANTICIPATING THE FUTURE OF SUSTAINABILITY IN QATAR: TOWARDS A "ROAD MAP” FOR MEANINGFUL STAKEHOLDER CONSULTATION ENGAGEMENT IN PLANNING THE RURAL SETTLEMENTS
}

\author{
ELGRITLY N. MAHMOUD \\ Urban Planning Department, Ministry of Municipality \& Environment, Qatar
}

\begin{abstract}
This paper attempts to focus on how planners and other stakeholders can assist the government' efforts towards preparing and implementing the Rural Settlements and Villages Planning projects through adopting the Stakeholders Consultation Engagement Approach in the context of this conference's main theme (Sustainable Development and Planning). For this purpose, the importance of this paper resides in facing the challenges related to achieving real sustainable development for the villages (and its surrounding rural settlements) to satisfy their future needs and aspirations for community facilities, infrastructure utilities and economic activities so as to become productive and self-contained entities. To meet the challenge mentioned above, this paper will highlight the lessons and experiences gained from best practice in the field of Stakeholders Consultation Engagement. Moreover, it seeks to identify the positive impacts of such lessons and experiences so as to apply them on Tenbik village case study. Keywords: sustainability, stakeholders consultation engagement approach, rural settlements planning, Qatar - Doha, Tenbik village, Qatar National Master Plan - QNMP2032, Qatar National Vision 2030 - QNV2030.
\end{abstract}

\section{INTRODUCTION}

Over the last few decades, Qatar has experienced a rapid growth in the urban areas, which has brought about fundamental changes, not only to the physical built environment, but also to people's perception, convention and cultural maturity. However, such urban expansion in the capital (Doha City) and its metropolitan region has happened at the expense of developing the rural settlements and villages that have become neglected and many of them have disappeared and become ruins. This in turn has led to the deterioration of the built environment and loss of quality of life in those villages. From this perspective, this paper attempts to focus on how planners and other stakeholders can assist the government' efforts towards the Rural Settlements and Villages Planning projects through adopting the Stakeholders Consultation Engagement Approach in the context of this conference main theme (Sustainable Development and Planning).

Hence, the paper will address the issue of delivering sustainable development in light of preparing a "Rural Settlements Development Plans Process" in Qatar particularly. For this purpose, the importance of this paper resides in facing the challenges related to achieving real sustainable development for the villages (and its surrounding rural settlements) to satisfy their future needs and aspirations for community facilities, infrastructure utilities and economic activities so as to become productive and self-contained entities.

To meet this challenge, this paper will present the concept of the "Stakeholders Consultation Engagement Approach" that has been partially adopted in examining the case study of Tenbik Village in Qatar [1]. Additionally, it highlights the lessons and experiences gained from best practice in the field of Stakeholders Consultation Engagement. Moreover, it seeks to identify the positive impacts of such lessons and experiences so as to apply them on Tenbik Case Study. 
This paper is divided into five main parts, the Section 1 provides a synopsis of the QNMP project, its components, its relationship with the rural settlements and villages planning and its adoption of Stakeholders Consultation Engagement in all its phases. The Section 2 sets out the justifications for adopting the pattern of villages and rural settlements development in the Gulf States and Qatar in particular.

Section 3 focuses upon the case study presented in this paper as a pilot project (planning and development of TENBIK village) in adopting the consultation and community engagement with the concerned stakeholders [1]. Such efforts attract the attention of the decision makers in the Ministry MME and set up a ministerial committee to formulate the basics of National Development Strategy for rural settlements and villages of Qatar [2]. Also, it highlights the case study that is presented in this research paper as a model which adopts Stakeholders Consultation and Community Engagement during the preparation of future development plans for the villages.

Section 4 formulates a proposed framework to address the Stakeholders Consultation Engagement Approach that can be adopted in Gulf States and Qatar in particular. Finally Section 5 concludes by proposing a Road Map to a meaningful and integrated approach of the Stakeholders Consultation Engagement to deliver sustainable development for Qatari Rural Settlements and Villages.

\section{INTRODUCING QATAR NATIONAL MASTER PLAN PROJECT FOR QATAR}

This section will be elaborated in the PowerPoint presentation that will be presented by the author of this paper next September 2018, it will highlight Qatar in figures (geographical location - area - population - progress in the areas of socio-economic development during the past two decades: from 2000 up to date). The scope of this part is to provide a Synopsis of Qatar National Master Plan Project - QNMP, in addition to identify the relationship between the QNMP Project with both Qatar National Vision (QNV2030) and the National Development Strategy (NDS). Moreover, it will elaborate the QNMP Project key elements which are: Qatar National Development Framework (QNDF); Municipal Spatial Development Plans (MSDP) and Urban Centers.

This section attempts also to highlight the Strategic objectives, policies and Policy Actions which are related to the planning of Rural Settlements and Villages in the context of QNMP Project. Finally, this part will come up with the adoption of the Stakeholders Consultation Approach during the preparation of all stages of the QNMP Project through conducting Public Opinion Survey and Consultation Sessions with the concerned Stakeholders during the preparation of QNDF and MSDP in addition to some sectoral strategies such as housing Strategy and Qatar Urban Planning Legislation.

\section{JUSTIFICATIONS FOR ADOPTING THE VILLAGES AND RURAL SETTLEMENTS PLANNING PATTERN IN THE GULF STATES AND QATAR IN PARTICULAR}

The urban scenery in the Gulf States is characterized by five main phenomena as follows [1].

\subsection{The dominant and prevailing cities}

Where the activities, facilities and various events are concentrated in such cities (such as sports, artistic and shopping events). As a result, rapid development occurs in all related fields, which is in turn reflected in the high rates of urban development at the expense of the rural areas and villages' development. That's why low rates of development prevail in these communities. On the other hand, such cities face many problems and challenges, perhaps the 
most important of which is the high rate of pollution (in all its forms), congestion and traffic jams (especially at peak hours), and the high cost of living standards.

\subsection{Swallowing of large cities for surrounding rural communities and villages}

In the early days of the oil era and unprecedented high returns and revenues, the Gulf governments implemented mega projects and pumped huge investments to establish these projects and services especially in the capitals and other major cities. This in turn resulting in socio-economic, and development gap between these cities and villages which are shrinking at a steady rate to continue their displacement and migration to major cities and capitals in search of better employment opportunities as well as high level of living standards and outstanding level of utility services and community facilities.

\subsection{Removing the features of the cultural heritage}

During the period of financial and economic prosperity experienced by the Gulf States, many areas and neighborhoods of traditional cities have been demolished. Instead of adopting policies to improve and upgrade the built environment, the demolition policy dominated through shovels and bulldozers both in urban and rural communities in that period. The absence of a strategic vision to preserve the cultural and historical heritage, and the lack of awareness among the decision makers of the importance of heritage and historical monuments in strengthening the loyalty of the citizen with his local customs, values and traditions.

\subsection{The loss of architectural and urban form identity for capitals and major cities}

The crisis of architectural and urban form identity in the main cities of the Gulf States has been reflected on high rates of development at an amazing speed. This in turn affected negatively on the continuity of the urban structure form between present and past, where exotic patterns of urban development and architectural styles were imported from the West. It is a replica of the Western world and its embodiment in most of the Gulf States. This situation has resulted in a monstrous and metamorphosis identity, under which the local identity of the urban and architectural product has been produced and implemented in most of the Gulf States.

\subsection{The Gulf citizen's sense of alienation and expatriation within his country}

In light of the above, the accumulation of such feelings can be justified and become firmly settled in the conscience of the Gulf citizen for two main reasons: firstly, attracting expatriates (at different levels of socio-economic, cultural and educational backgrounds) as a result of the economic boom witnessed by the Gulf States in mid of the last century. Secondly, Migration and continuous population displacement of villages and rural communities for settling down in capitals and major cities.

Thus, a society that lacked socio-economic and cultural cohesion emerged. The Gulf society then transferred from a type of extended family to independent nuclear families, which negatively affected the deepening of the feeling of alienation and expatriation. In order to limit the phenomena described above, the adoption of an integrated strategy for the development of rural communities and villages should be covered from the physical, environmental and socio-economic perspectives. This will result in several positive returns, perhaps the most important of which are: achieving comprehensive development throughout 
the country (urban and desert/rural areas), as well as reducing the developmental and demographic gap between urban and rural areas.

In this context, we can identify the reasons and justifications for planning and developing these settlements and villages as follows: Firstly, the value of these rural settlements in maintaining and preserving the local identity. Secondly, the inclusion and containment of such villages to some archaeological and heritage sites. Thirdly, fear of those villages from becoming extinct and ruin. Fourthly, maintain a traditional lifestyle that reflects the depth of traditions and conservative societies [3].

\section{CASE STUDY: PLANNING OF TENBIK VILLAGE AS A PILOT PROJECT}

\subsection{Reasons and justifications for choosing the case study: Tenbik village}

One of the most important reasons for selecting the case study is the adoption of two main mechanisms during the preparation of planning studies for this project: the first one is the Coordination and consultation with the related parties: through conducting consultation sessions and meetings with the concerned stakeholders. Secondly, the co-existence with the actual living reality of the planning project that we are studying.

In addition, a teamwork was formed to undertake this project, which led to the contribution of formulating an integrated and realistic vision for the project. In addition, resolving planning decisions embodied in the proposed Master Plan to ensure the achievement of THREE main goals: the feasibility of various elements of the project, satisfying the local community' needs and desires and accomplishing the consistency with the guidance and directions of the spatial development plan of the municipality (within which the target village to be studied).

During the consultation sessions conducted with the concerned parties involved in the implementation of the road and infrastructure networks within the framework of the Municipal Spatial Development Plans - (MSDP), the project consultant, who has been assigned the planning and design of road and infrastructure networks and Landscaping for Tenbik village, presented the obstacles that prevent and hinder the implementation of the project, and then he introduced two main alternatives:

First alternative: The project is designed and implemented according to the Land Use Plan approved by the Urban Planning Department - (which is not currently updated).

Second alternative: A new master plan needs to be prepared to meet and satisfy the expected future requirements of the urban growth for Tenbik village in a context consistent with the spatial development plan of Al Daayen Municipality. This alternative has been agreed to be adopted [1].

\subsection{An example of the Stakeholders Consultation Engagement adoption}

In light of the consensus regarding the adoption of the second alternative, series of meetings and consultation sessions were held among the various representatives of the QNMP Project, Urban Planning Department, Al Daayen Municipality and the consulting firm (that has been assigned the responsibility of planning and designing the project elements). Such meetings and sessions have come up with a meeting held in the municipality's headquarters in the presence of representatives of the local community for the village.

The consultations and meetings led to an agreement on decisions that were reflected in the proposed Master Plan as follows: extending the scope of the consultant's tasks, coordination with related agency "Public Works Authority" (through which the consultant 
works) to postpone the implementation of the project until the preparation and approval of the future Master Plan, having an agreement on preparing the future Master Plan for Tenbik, and obtaining the agreement on the Site and area for many land uses and activities that have been allocated in the proposed Master Plan.

\subsection{The proposed methodology for the study}

An integrated methodology has been adopted including planning and implementation of this project. It is consisting of five main phases: the first phase is related to studying the current situation and collecting the relevant data and information. The second one unveils a thorough analysis of the existing studies (constraints, problems, opportunities). This phase has been concluded with the most important findings and indicators upon which the master plan for the village to be prepared. The third stage is proposing alternatives, evaluating them and choosing the most appropriate one. The fourth has to do with preparing detailed studies of the proposed Master Plan of the village. The fifth and last stage is concerned with formulating the project implementation system, which depends upon two pillars: firstly: identifying the project priorities for the future village development. Whereas secondly: holding consultation sessions with the concerned stakeholders and engaging them into all stages of project implementation process [3].

\subsection{The fundamental outputs and recommendations of the study}

An integrated concept of the most important outputs has been formulated for the project, including the following: study report, future development plan of the village and its surroundings, proposed Master Plan of the village, implementation phases of the proposed plan, zoning map and 3D Model of the proposed Master / Development Plan.

The study was concluded by a list of recommendations - that can be summarized as follows. Firstly: supporting and encouraging the farm owners and motivating them to develop agricultural and livestock production. Secondly: setting up a complex of non-polluting craft activities. Thirdly: restoration and upgrading the heritage site of the old village and exploiting it as a tourist destination. Fourthly: setting up of a permanent committee of the concerned stakeholders including representatives of local community, in order to enhance the "participatory planning" approach by conducting consultation sessions in all phases of project planning and implementation [1].

\subsection{The proposed recommendations of the Ministerial Committee entrusted with developing a National Development Strategy for rural settlements and villages of Qatar}

The planning study of Tenbik Village Case drew attention to the importance of formulating a national strategy for the development of villages and rural settlements at the state level. This led the HE the minister of MME (Ministry of Municipality and Environment) to form a ministerial committee to set out the outline of the strategy. This committee conducted (8) meetings within (2) months and has been concluded with four main recommendations: the first one: identifying a priority list of the most important villages and rural communities to be planned and developed either at immediate or in the short or medium term). The second is concerned with monitoring and observing a list of issues to be discussed, resolved and agreed upon by the Committee. It worth to point out that there was a consensus among the committee members in respect to the importance of adopting the Participatory Planning 
Approach through conducting consultation sessions with all concerned stakeholders, including representatives of the local community.

Whereas the third recommendation has to do with formulating suggestions and mechanisms to deal with the problems and issues identified in the previous item, for example: the problem of marginalizing the role of local community and the concerned stakeholders in the future development planning studies of villages and rural communities. It was recommended to design a questionnaire form and conducting interviews with the local community representatives so that their needs, problems, desires and wishes can be identified in planning the future of their village.

The fourth set of recommendation and proposals formulated by the abovementioned committee consists of two sets:

The first group concerned with the formulation of suggestions dealing with problems and issues related to the planning and development of villages and rural settlements along Qatar. The most important one is maximizing the role of the targeted local community and engaging their representatives (elected members of the Central Municipal Council - CMC) as well as the dignitaries and the elite of the community in addition to the civil society institutions operating in the village.

The second set of recommendations related to the planning aspects of implementing the Committee's proposals. They highlighted the importance of community participation and consultation with the concerned stakeholders including the local community in all phases of the project study. The planning study report includes a specialized chapter that documents the process of Stakeholders Consultation Engagement (including the local community representatives) to consolidate their role in shaping the vision and strategy of the future development planning for their communities [2].

\section{FORMULATING A PROPOSED ROADMAP TO ADOPT STAKEHOLDERS CONSULTATION ENGAGEMENT APPROACH TO ACHIEVE A SUSTAINABLE DEVELOPMENT FOR RURAL SETTLEMENTS IN QATAR}

This section will develop a proposed road map (suggested by this research) to adopt the approach of Consultation and Community Engagement in a context consistent with traditions and customs of the Gulf society. It should be noted that the formulation of the proposed roadmap was based on four main pillars

\subsection{United Nations goals and objectives for sustainable development 2030}

The Sustainable Development Goals (SDGs) are a new set of goals, targets, and indicators published by the United Nations to define the global development priorities for the 2015 2030 period. The SDGs are the outcome of the largest consultation process in UN history, the UN created an open working group with representatives from 70 countries to develop a draft agenda. The UN also launched a series of "global conversations" - 11 thematic and 83 national consultations with governments, civil society institutions, activists, academics, the private sector, others; a door-to-door surveys - and an online "My World" survey, which asked people to identify what they would like to see in the 2030 Agenda for Sustainable Development, which lays out its 17 goals and 169 targets, or sub-goals [4].

The UN preamble for the SDGs, emphasizes clearly that civil society groups have a role to play in both the implementation and the monitoring of activities related to the new global agenda. Therefore, this UN document has given public policy advocates and the civil society a forum or the platform through which to speak with a clear and legitimate voice. This in 
turn, has resulted in strengthening the relationships between the public, government agencies and the civil society institutions.

Goal No (11) that is related to make human settlements inclusive, safe, resilient and sustainable - is solely dedicated to the future of cities and their inhabitants through: ensuring access for all to adequate, safe and affordable housing and basic services, providing access to safe, affordable and sustainable transport systems for all, improving road safety, notably by expanding public transport. In addition to adopting the participatory, integrated and sustainable human settlements planning and management and strengthening efforts to protect and safeguard the local cultural and natural heritage. Such goal is also dedicated to providing access to safe, inclusive and accessible public spaces for all classes and segments of society in particular for women, children, aged people and persons with disabilities.

Thus, goal \#11 advocates the right of all the inhabitants to live in decent conditions and to shape the future of their built environment. This concept based upon community participation and in the belief that officials whether they are elected or non-elected should satisfy the needs and aspirations of all members of the local society. In defending the Right to the City, we not only promote a future of equity and sustainability, but also lays the groundwork for a new pattern of relationship among government, the private sector, and civil society.

The UN General Assembly unanimously adopted Resolution \#1/70, and this nearly universal endorsement imbues the goals with an authority upon which nations and citizens alike can build a plan to achieve at least some of the SDG's grand promise [4].

\subsection{The New Urban Agenda 2016 - NUA16}

The New Urban Agenda was adopted at the United Nations Conference on Housing and Sustainable Urban Development (Habitat III) in Quito, Ecuador, on 20 October 2016. It was endorsed by the United Nations General Assembly at its sixty-eighth plenary meeting of the seventy-first session on 23 December 2016 [5].

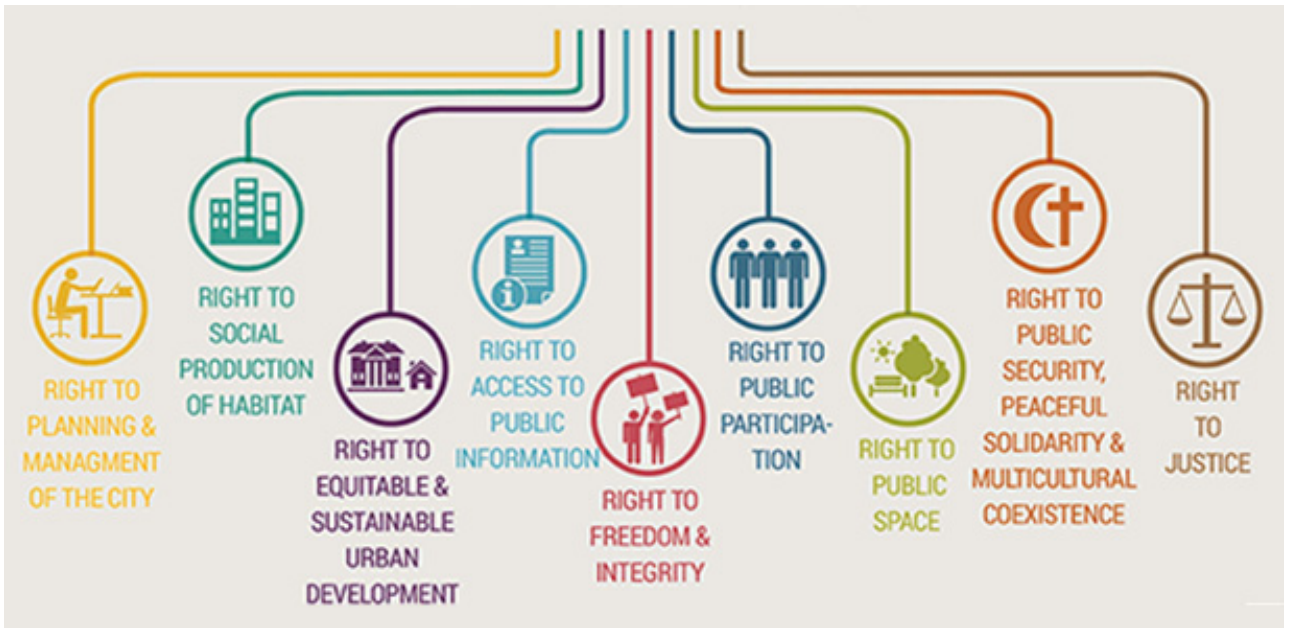

Figure 1: Rights of citizenship and participation in the planning and management of the city. (Source: United Nations Sustainable Development Goals 2030 (2016). Page (4) World Charter for the Right to the City (2005). Graphic by Tadamun (2015).) 
The NUA16 represents a shared vision for a better and more sustainable future - one in which all people have equal rights and access to the benefits and opportunities that cities can offer. Thus, this agenda aims at adopting the human settlements for a participatory approach in a way that enhances social relations.

\subsubsection{NUA16 Principles and commitments}

Those principles and commitments will be discussed from the extent of adopting the participatory planning approach which state upon FOUR main principles: the first one: ensure community participation is secured by providing all infrastructure and community facilities as well as basic services to all segments of society on an equal basis. Secondly, develop appropriate urban policies and implement them within partnerships at the national and local levels, among the concerned stakeholders, as well as enhance synergies between all government agencies at all levels so as to achieve sustainable and integrated urban development.

Thirdly, strengthen and enhance the management of human communities by establishing an institutional system and mechanisms that empower the stakeholders to include all layers of the concerned bodies. In addition to provide public open space for community engagement, economic growth and the protection of environmental resources in a comprehensive and sustainable manner for all and allows all segments of society to participate effectively in planning decision-making processes and follow-up implementation. Fourthly, encourage the provision of open and green spaces characterized by the availability of safety and quality factors, so that spaces to be allocated for stimulating social interaction, providing economic exchange and encouraging dialogue between the various groups of society and culture. In addition to set up participatory communities that Promote Connectivity, Community Engagement and maximize the use of natural and cultural heritage in order to promote community participation.

\subsubsection{Setting up a coherent Urban Governance Framework}

This can be done through the following elements: first and foremost, formulating inclusive, implementable and participatory urban policies. the second component adopting transparent and accountable mechanisms. The third one is related to supporting the local governments in determining their own administrative and management structures, in line with national legislation and policies, as appropriate, in order to adapt to local needs.

The fourth element of the framework is concerned with encouraging appropriate regulatory frameworks and support to local governments in partnering with communities, civil society institutions and the private sector to develop and manage basic services and infrastructure networks, ensuring that the public interest is preserved. The fifth and final component has to do with promoting participatory approaches at all stages of the urban and territorial policy and planning processes, from conceptualization to design, budgeting, implementation, evaluation and review. In addition to creating new forms of direct partnership between governments at all levels and civil society, including through broadbased and well-resourced permanent mechanisms and platforms for cooperation and consultation open to all, using information and communication technologies [5].

\subsubsection{Adopting innovative mechanisms and means of implementation}

This can be done through the following: firstly, enhancing partnerships among Governments at all levels, the private sector, civil society, and other concerned actors. Secondly, promoting the capacity of local governments, to work with All segments of the society: including women, children, youth, the elderly, persons with disabilities, and local communities. In 
addition to the civil society, academia and research institutions in shaping and formulating the organizational and institutional governance processes, enabling them to participate effectively in decision-making Planning process regarding urban and rural development.

Thirdly, fostering the creation and establishment of open, user-friendly and participatory data platforms and share knowledge among local governments and the concerned stakeholders. This in turn will lead to enhance effective urban planning and management, efficiency and transparency through e-governance, approaches assisted by information and communication technologies.

\subsection{Paper titled "Stakeholder Engagement a RoadMap to Meaningful Engagement"}

This paper aims to provide an understanding of, and practical tips for, successful stakeholder engagement. It focuses particularly on engaging communities, NGOs and wider civil society institutions. It is written by a Doughty Centre Associate Neil Jeffery who has had extensive experience as an NGO campaigner and director; and more recently, as an adviser to a number of Fortune 500 corporations on engaging stakeholders [6].

\subsubsection{The process flow of stakeholder engagement}

The third section of the abovementioned paper provides detail and examples of each process. In brief, we can summarize this process as follows:

Stage 1, planning: Identify our key objectives, issues to be addressed and the stakeholders we prioritize as critical to our organization and suit the type of the project.

Stage 2, understand our stakeholders: Identify the urgency they feel for their issues, the legitimacy of their interest and the power they have to impact on our organization. Understand their wants and needs and how this correlates with our project target. This in turn, will help us to profile the priority stakeholders.

Stage 3, preparing internally to engage: Dedicate appropriate time and resources to identify possible commonalties between our organisation and the concerned stakeholders. This requires to have an agreement on the commitment that our organisation will give to stakeholder engagement and the process.

Stage 4, building trust: Different stakeholders will come with different levels of trust and willingness to trust. This will require us to interact with their trust level, and to adapt to the level of trust present and needed.

Stage 5, consultation: For overall success it is important to achieve the following through the consultation process: first and foremost, is the fair representation of all stakeholders. The second is being responsive by providing information and proposal that respond directly to their expectations and interest previously identified. The third has to do with contextualizing information so that stakeholders get a detailed, holistic picture. The fourth is concerned with providing complete background information stakeholders need to draw reasonable conclusions. The fifth but the last has to be realistic in negotiations with possible trade-off of expectations, needs and objectives. This in turn will help achieve agreement and build trust. Furthermore, it is necessary to emphasize that the mechanism of consultation includes personal interviews, workshops, focus groups, public meetings, surveys, participatory tools and stakeholder panels. We should choose the most relevant mechanisms for each stakeholder group as one template won't fit all. We have to prioritizing issues from our and the stakeholders' viewpoint, as well as understanding the stakeholders' issues of importance.

Stage 6, respond and implement: we should decide on a course of action for each issue agreed upon - understanding possible stakeholder reactions to our proposal will help us to develop a more successful proposal of action, and this will enable us to respond successfully 
to their needs, wants, desires and concerns. A process is provided offering guidance on how to implement measures for the agreed issues identified.

Stage 7, monitor, evaluate and document: Knowledge management is critical for capturing information and sharing what is learned. Transparency of the process is greatly helpful by accurate documentation, especially if our organization reports on Stakeholder Consultation Engagement. We should report back to stakeholders on progress, in a form and language appropriate to them [6].

\subsubsection{Golden Rules' that will encourage and foster meaningful Stakeholders Consultation Engagement}

Such rules can be summarized in four main points: firstly, building trust with stakeholders aided by understanding their viewpoints and motivations. Secondly, understanding and being transparent about the motivation of both stakeholders and our organization can help overcome differences. However, understanding and articulating this can help close this gap. Thirdly, assessing past non-productive or unsuccessful stakeholder consultation and community engagement behavior will help the organization to learn from past experiences. Fourthly, considering four vital influences on our stakeholder engagement efforts: organizational culture, structure, human resources and learning - that will be explained in the next point.

\subsubsection{Recommendations towards a meaningful stakeholder}

The concept of meaningful stakeholder engagement goes far beyond the idea of simple contact or coordination inherent in crisis management or consultation as seen in stakeholder management; inherent in the concept of meaningful engagement. There are THREE main points need to be highlighted: the first is a two-way process should be adopted: including exchange of information, research, analysis and opinions between the planning organization and stakeholders. The second is related to the commitment and willingness from each side to contemplating changes in behavior, actions, priorities, organizational structure, staffing and training product development. The third has to do with the actions based on an unambiguous and clear set of objectives, with clearly defined outputs, and projected outcomes and impacts.

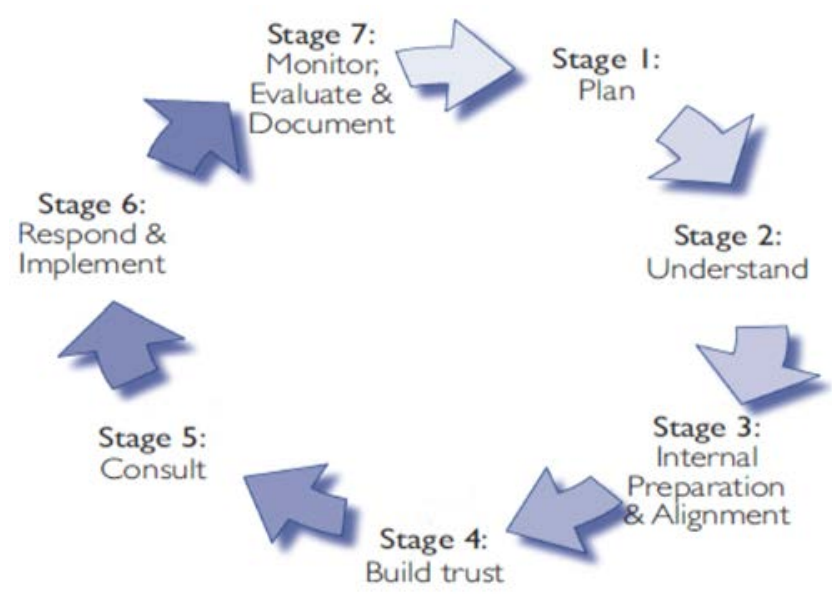

Figure 2: The process flow of stakeholder engagement. (Source: Smith, 1989.) 
There are number of considerations upon which any planning firm or organization should reflect during conducting the Stakeholders Consultation Engagement process. The considerations either outline strategies to be implemented or items to be addressed, as a means to maximize the impact and success of meaningful stakeholder relations. The recommendations are classified according to their relevance to organizational culture, organizational structure, organizational human resources, and organizational learning. They can be briefly outlined as follows [6]:

- Organizational culture: Ensure that successful Stakeholders Consultation Engagement - SCE behavior is embedded throughout all layers of the organization and is reflected and supported by organizational culture. In addition, appropriate leadership from the very top of the organization is needed to demonstrate real commitment to SCE, not only through words and declarations, but also through real actions. In this context, senior management should get involved in the wider debate about how society builds strong institutions and overcomes challenges as a way of demonstrating commitment. Also, it is important for managers to maintain a dialogue with those they disagree and maintain a position of active negotiation with them i.e. be willing to concede on issues as a way of making progress. Finally, the staff should be encouraged to understand and develop their thinking regarding the motivation for conducting SCE.

- Organizational structure: The senior management of the planning organization should clearly demonstrate that SCE is regarded as a strong facilitator of its organization's success. By this view, successful SCE should be seen as a strategic function and not just a communications or coordination task as it provides value for, and feeds into, all tasks and responsibilities of the organization. Therefore, building the capacity of the staff organization to better empathize with stakeholders; will help to grow the appreciation for stakeholder motivations and objectives.

- Organizational human resources: It is a paramount important to continuously develop in-house skills of the organization staff and expose them to unfamiliar environments. This in turn will ensure that they stay up-to-date with fast changing necessities regarding SCE. However, hiring staff from stakeholder organizations, or investing in secondments to/from stakeholders' organizations, is considered one of the best ways to improve knowledge and understanding of stakeholders and their priorities. Therefore, it is necessary to allocate a budget for strengthening the internal SCE capacity through setting out training programs as a cost-effective mechanism to mitigate risk

- Organizational learning: In the outset, appropriate training programs should be provided for staff to support and promote their performance and capacities in respect to conduct successful SCE. This will be resulted in enhancing the ability of all staff and enable them to conduct analysis regarding stakeholder motivations, objectives and value. This in turn will ensure that knowledge and learning is captured and shared across the organization. As a result, this will guarantee that advances and activities are retained, shared and transmitted to additional staff [6].

\subsection{Proposed roadmap model to adopt a sustainable process for meaningful Stakeholder Consultation Engagement in the context of Qatar}

In light of Sections 4 and 5 of this paper, a Roadmap Model for SCE can be formulated to suit the context of the Gulf States in general and Qatar in particular. This will be elaborated 
in the oral presentation of the research at the Sustainable Development and Planning Conference that takes place in September 2018, in Siena, Italy

\section{REFERENCES}

[1] Ministry of Municipality and Urban Planning - MMUP, Directive Plans of villages \& rural settlements development project for Qatar, Qatar, Doha. Unpublished Report: QNMP Project - MMUP, 2015.

[2] Ministry of Municipality and Environment - MME, Final Recommendations of the Ministerial Committee for planning villages and rural settlements in outside Metropolitan Doha, Qatar, Doha. Unpublished Report: QNMP Project - MME, 2017.

[3] Ministry of Municipality and Urban Planning - MMUP, Towards the formulation of national strategy to satisfy the basic needs of villages and rural settlements in Qatar, Qatar, Doha. Unpublished Report: QNMP Project - MMUP, 2011.

[4] United Nations Sustainable Development Goals 2030, A user's guide to the sustainable development goals, Egypt, Cairo. UN General Assembly, 2016.

[5] United Nations Conference on Housing \& Sustainable Urban Development, New Urban Agenda, Quito, Ecuador, The Habitat III Secretariat, 2017.

[6] Jeffery, N., Stakeholder engagement: A road map to meaningful engagement, Doughty Centre, Cranfield School of Management, 2009.

[7] Hassan Atef, H., City planning in Qatar, Qatar, Doha. Dar El Oloum - Qatar University, 1994. 